\title{
Correlative X-ray and Electron Spectroscopy of the Materials-Biology Interface Reveals Insights into Impact of Engineered Nanomaterials on Human Physiology
}

\author{
Alexandra E. Porter \\ Department of Materials and London Centre for Nanotechnology, Imperial College London, UK
}

Demand for silver nanomaterials is increasing rapidly in electrical and mechanical and in health and medical applications due to their thermal, electrical conductive and other properties. The continued commercial up-scaling of silver nanomaterial production and application needs to be accompanied by an understanding of the occupational health, public safety and environmental implications of these materials. There have been numerous in vitro studies and some in vivo studies of silver toxicity; however, the results of these studies are frequently inconclusive. Some of the variability between studies has arisen due to the propensity for the physicochemistry of the silver nanomaterials to alter, depending on the environment they encounter, which can alter their bioreactivity with proteins, cells and tissues. For this reason, it is important to accurately characterise the materials before, and at the point of exposure, and at the nanomaterial-tissue interface, to appreciate their impact on human health. However, accurate spatially resolved analyses of these interfaces require thorough characterisation and spatially resolved imaging and analysis under complex, dynamic experimental conditions. The aim of this work is to apply correlative imaging and analysis techniques to characterise the chemistry of the silver nanomaterials and probe these interfaces across a range of length scales. Nanoanalysis in the aberration corrected transmission electron microscope has been correlated to in situ studies of the Ag-protein and Ag-cell interface, using x-ray scattering and confocal microscopy. Specifically, the mechanisms by which the physicochemical properties (i.e. aggregation state, morphology and chemistry) of the Ag change in various biological milieu (i.e. relevant proteins, cell culture media, lipids and other molecules, and biofluids), and affect subsequent interactions with and within lipids, cells and tissues, has been monitored.

Using a combination of in-situ scattering and spectroscopic methods, coupled with high resolution imaging, we have demonstrated that the rate of $\mathrm{Ag}^{+}$ion release from $20 \mathrm{~nm}$ diameter citrate capped silver nanoparticles (AgNPs) is strongly pH-dependent [1]. As predicted, the Ag nanoparticles dissolved in acidic $\mathrm{pH}$, whereas they were comparatively stable at extracellular $\mathrm{pH}$. However, the amount Ag+ dissolution is strongly dependent on the cell culture media. In particular, there is a strong interaction with S containing groups of ionic salts, although we observe that the Ag nanowires did not readily sulphidise in media containing sulphur-rich cysteine [2]. DPPC, a phospholipid which is the primary component of lung lining fluid, delayed the release of $\mathrm{Ag}+$ ions, but did not significantly alter the total amount of $\mathrm{Ag}+$ released from the AgNPs after two weeks. In addition, DPPC was found to improve the dispersion of the AgNPs, inhibiting aggregation and coarsening. Transmission electron microscopy (TEM) images revealed that the AgNPs become coated with a DPPC layer serving as a semi-permeable protective layer. Hence, we have shown that lung lining fluid, and particularly DPPC can modify the particle aggregation state and the kinetics of $\mathrm{Ag}^{+}$ion release of $\mathrm{AgNPs}$ inhaled into the lung.

The biostability and distribution of both AgNPs and Ag nanowires (AgNWs) were characterised as a function of time, following incubation of the Ag nanowires with epithelial cells. [3] TEM imaging and 
analysis, combined with confocal microscopy of live cells, demonstrated that the AgNWs penetrated through the plasma membrane of type 1 epithelial cells and distributed within the cytoplasm and endosomes. The AgNWs were dissolved inside the cells and subsequently transformed into highly insoluble $\mathrm{Ag}_{2} \mathrm{~S}$. We suggest that transformation of the nanowires to $\mathrm{Ag}_{2} \mathrm{~S}$ acts as a $\mathrm{Ag}^{+}$ion 'trap' that will significantly limit the short-term nano-Ag toxicity and their impact on the environment and lung physiology.

[1] Leo, B.F., Chen, S., Kyo, Y., et al., Environ. Sci. Technol, 47, 19 (2013) p. 11232

[2] Chen, S., Theodorou, I.G., Goode, A.E., et al., Environ. Sci. Technol, 3,47(23) (2013) p.13813

[3] Chen, S., Goode, A.E., Sweeney, S., et al., Nanoscale, 21; 5(20) (2013) p. 9839-47

4] The authors acknowledge the Ministry of Higher Education of Malaysia, the NIEHS (grant number U19ES019536) and the US EPA/NERC (EPA STAR RD83469301 and NERC), an ERC starting grant (Project number 257182, CNTBBB) and the Leverhulme Trust.

Dr. D. Botelho, Miss B. Leo, Dr. C. Massa, Dr. S. Sarkar, Dr. D. McPhail., Prof. T.D. Tetley, Prof. Kain Fan Chung, 4, Dr. S. Chen1, Prof. J. Zhang, Prof. S. Schwander, Prof. M. Shaffer, Prof. A. Gow. Dr. Karin Muller, Dr. Jeremy Skepper and Prof. M.P. Ryan are thanked for their scientific and experimental contributions to the work. 